DESY-07-208

YITP-SB-07-33

\title{
Tensor calculus for supergravity on a manifold with boundary
}

\author{
Dmitry V. Belyaev \\ Deutsches Elektronen-Synchrotron, DESY-Theory \\ Notkestrasse 85, 22603 Hamburg, Germany \\ E-mail: dmitry.belyaev@desy.de

\section{Peter van Nieuwenhuizen} \\ C. N. Yang Institute for Theoretical Physics, SUNY at Stony Brook \\ Stony Brook, NY 11794-3840, USA \\ E-mail: vannieu@max2.physics.sunysb.edu
}

\begin{abstract}
Using the simple setting of 3D $N=1$ supergravity, we show how the tensor calculus of supergravity can be extended to manifolds with boundary. We present an extension of the standard $F$-density formula which yields supersymmetric bulk-plus-boundary actions. To construct additional separately supersymmetric boundary actions, we decompose bulk supergravity and bulk matter multiplets into co-dimension one submultiplets. As an illustration we obtain the supersymmetric extension of the York-Gibbons-Hawking extrinsic curvature boundary term. We emphasize that our construction does not require any boundary conditions on off-shell fields. This gives a significant improvement over the existing orbifold supergravity tensor calculus.
\end{abstract}




\section{Contents}

1. Introduction 1

2. Co-dimension one gauge algebra

$2.13 \mathrm{D} N=1$ gauge algebra

2.2 Einstein boundary condition

2.3 The unbroken half of bulk susy 5

2.4 Modified $\epsilon_{+}$susy 6

2.5 The reduced gauge algebra 6

3. Co-dimension one submultiplets 7

$3.13 \mathrm{D}$ supergravity multiplet

3.2 Co-dimension one split 8

In.3 Induced supergravity multiplet 9

3.4 Radion multiplet 10

3.5 Extrinsic curvature multiplet

3.6 Submultiplets of the 3D scalar multiplet 11

3.7 Separately susy boundary actions 12

1. Susy bulk-plus-boundary actions

4.1 The " $F+A$ " formula

4.2 Extended $F$-density 13

4.3 Super-York-Gibbons-Hawking construction 14

5. Summary and Conclusions $\quad 16$

\section{Introduction}

Supersymmetry (susy) and supergravity (sugra) were first formulated in the 1970's as field theories in $x$-space (the $x$-space or component approach). A tensor calculus for 4D $N=1$ rigid susy, with Poincaré or conformal symmetries, was developed by Julius Wess and Bruno Zumino in their pioneering work [1]. For local susy (sugra), a tensor calculus for 4D $N=1$ models was obtained in [2, 3]. At the same time, the superspace approach of Salam and Strathdee [4] was extended to supergravity by Wess and Zumino [5] and was shown to be equivalent to the $x$-space tensor calculus approach [6]. Both approaches have been used since, and each has its own virtues. 
In all these studies, boundary effects were mostly ignored and various total derivatives were simply dropped. Already in the $x$-space approach, one calls a Lagrangian supersymmetric if its susy variation is a total derivative. In superspace, manipulations with susy-covariant derivatives $D_{\alpha}$ often produce total $x$-space derivatives which are again discarded under the $x$-space integration. One cannot do so in the presence of boundaries in $x$-space, which is why the superspace and tensor calculus approaches are not obviously extendable to a manifold with boundary.

Susy models in the presence of $x$-space boundaries have been studied before. Boundary terms for open fermionic strings [7] and the Casimir effect in 4D susy theories [8] were among the first considered. (For a flavor of other models discussed over the years, see [9].) Already in [7] it was argued that one needs boundary conditions (BC) to maintain (at least part of) susy in the presence of a boundary, and that the BC must, in turn, be left invariant under susy transformations (that is, form a "susy orbit" [10]). This approach, which we will call "susy with BC," was used in most works on susy in the presence of boundaries.

In a recent analysis of [10, 11], the $\mathrm{BC}$ required by the Euler-Lagrange variational principle, were considered together with the $\mathrm{BC}$ needed to maintain susy of the actions. The orbit of all BC was constructed, and the functional space of off-shell fields was defined by the set of all constraints. Here we take a completely opposite point of view: we develop an approach to rigid and local susy in which off-shell fields are totally unconstrained.

Our approach gives classical ${ }^{1}$ bulk-plus-boundary actions that are susy (under a half of bulk susy) without using any BC on fields. We call our approach "susy without BC" to contrast it with the "susy with BC" approach used so far. ${ }^{2}$ For rigid susy, the validity of this approach has already been established by one of us in 13. The key ingredient used there, which made the construction particularly simple, was the co-dimension one decomposition of (rigid) superfields [14]. In this article, we will give a first complete realization of this approach in the case of local susy (sugra). We restrict our discussion to a 3D space-time and show how the complete tensor calculus for 3D $N=1$ local susy can be extended to take boundaries into account. Co-dimension one decomposition of the bulk susy multiplets will play an essential role in our construction. An extension of our construction to higher dimensions and its superspace realization will be discussed elsewhere [12].

Understanding supergravity on a manifold with boundary is an interesting mathematical problem. It is also important for various physical models that have appeared in the past decade. Notably, the 11D Horava-Witten (HW) construction [15] and the 5D RandallSundrum (RS) scenario [16] (whose minimal supersymmetrization was achieved in [17]). ${ }^{3}$ In

\footnotetext{
${ }^{1}$ At the quantum level, local susy is replaced by BRST symmetry, but the same approach can be followed [10, 11].

${ }^{2}$ We will impose $\mathrm{BC}$ on symmetry parameters, but not on fields. Of course, BC on fields follow upon applying the variational principle to our actions, but these $\mathrm{BC}$ are not needed in the proof of susy of the actions. Whether these BC form susy orbits [10, 11] is a separate issue that we will discuss elsewhere [12].

${ }^{3}$ The HW and (susy) RS models are usually discussed in the "upstairs picture" (on the $S^{1} / \mathbb{Z}_{2}$ orbifold). The alternative "downstairs picture" (on a manifold with boundary) approach to these models was considered, for example, in [18] and [19], respectively. Here we adhere to the "downstairs picture" description.
} 
these models, one starts from a (standard) bulk supergravity action and tries to construct a boundary action (involving, in general, additional boundary-localized fields) that makes the whole system supersymmetric (under a half of bulk susy, with the other half being spontaneously broken by the presence of the boundary). As of now, most approaches to constructing such susy bulk-plus-boundary actions have relied on certain approximations. For example,

1. the $11 \mathrm{D} \mathrm{HW}$ action is susy only to a certain order in the expansion parameter $\kappa^{2 / 3}$ [15, 18];

2. the 5D orbifold supergravity tensor calculus of [20, 21] relies on using standard orbifold "odd=0" BC which, in general, are incompatible with the $\mathrm{BC}$ one derives from the variational principle [22];

3. the 5D constructions of [23], which incorporate $\mathrm{BC}$ following from the variational principle, are worked out only to lowest fermi order.

We hope that our approach, which works without any approximations or assumptions, will help to bring these constructions to completion.

We base our construction on the existing tensor calculus for $3 \mathrm{D} N=1$ and $2 \mathrm{D} N=(1,0)$ supergravity. This tensor calculus was worked out by Uematsu [24, 25], following the 4D $N=1$ results of [2]. In these derivations, conformal sugra plays a fundamental role, but we consider only Poincaré sugra in this article.

Our construction will consist of the following steps.

First, we analyze the algebra of supergravity gauge transformations. We recall why, in the presence of a boundary, one can (typically) preserve only half of bulk susy, and prove that the restriction to this half of susy reduces the whole 3D $N=1$ gauge algebra to the standard 2D $N=(1,0)$ gauge algebra, without imposing any $\mathrm{BC}$ on fields. We note that the analysis becomes particularly simple in a special Lorentz gauge (which is opposite to the standard Kaluza-Klein choice) and we adopt that gauge from then on. As a consequence, the preserved half of susy transformations gets modified by a compensating Lorentz transformation.

Second, we perform a co-dimension one decomposition of the 3D supergravity tensor calculus. This gives, in particular, the induced supergravity multiplet that is necessary for constructing separately susy boundary actions. The decomposition does not rely on using any BC (like "odd=0" BC used in 20, 21) and is applicable to any hypersurface parallel to the boundary.

Third, we show that on a manifold with boundary, the standard 3D F-density formula must be extended by the addition of a boundary $A$-term. The extended $F$-density formula automatically gives bulk-plus-boundary actions that are susy (under the half of bulk susy) without using any $\mathrm{BC}$ on fields. We also write the extended $F$-density in terms of the codimension one submultiplets.

To illustrate the construction, we finally apply the extended $F$-density formula to the 3D $N=1$ scalar curvature multiplet. This will show that the minimal susy bulk-plus-boundary 
action, with the standard 3D $N=1$ supergravity in the bulk, does not include the YorkGibbons-Hawking term [26]. The latter comes as a part of a separately susy boundary action that one needs to add in order to relax field equations which would otherwise be too strong.

\section{Co-dimension one gauge algebra}

In this section, we will show how the $3 \mathrm{D} N=1$ supergravity gauge algebra ${ }^{4}$ reduces naturally to the $2 \mathrm{D} N=(1,0)$ supergravity gauge algebra on the boundary, as well as on co-dimension one slices parallel to the boundary.

\subsection{D $N=1$ gauge algebra}

The gauge transformations of the 3D $N=1$ (off-shell) Poincaré supergravity are the Einstein (general coordinate) transformation $\delta_{E}\left(\xi^{M}\right)$, the local Lorentz transformation $\delta_{L}\left(\lambda^{A B}\right)$ and the susy transformation $\delta_{Q}(\epsilon)$. The complete gauge algebra reads ${ }^{5}$

$$
\begin{gathered}
{\left[\delta_{E}\left(\xi_{1}^{M}\right)+\delta_{L}\left(\lambda_{1}^{A B}\right)+\delta_{Q}\left(\epsilon_{1}\right), \quad \delta_{E}\left(\xi_{2}^{M}\right)+\delta_{L}\left(\lambda_{2}^{A B}\right)+\delta_{Q}\left(\epsilon_{2}\right)\right]} \\
=\delta_{E}\left(\xi_{\mathrm{comp}}^{M}\right)+\delta_{L}\left(\lambda_{\mathrm{comp}}^{A B}\right)+\delta_{Q}\left(\epsilon_{\mathrm{comp}}\right)
\end{gathered}
$$

where the composite parameters are

$$
\begin{aligned}
\xi_{\text {comp }}^{M} & =2\left(\bar{\epsilon}_{2} \gamma^{M} \epsilon_{1}\right)+\left[\xi_{2}^{N} \partial_{N} \xi_{1}^{M}-(1 \leftrightarrow 2)\right] \\
\lambda_{\text {comp }}^{A B} & =2\left(\bar{\epsilon}_{2} \gamma^{N} \epsilon_{1}\right) \widehat{\omega}_{N}{ }^{A B}+\left(\bar{\epsilon}_{2} \gamma^{A B} \epsilon_{1}\right) S+\left[\xi_{2}^{N} \partial_{N} \lambda_{1}^{A B}+\lambda_{2}^{A} \lambda_{1}^{C B}-(1 \leftrightarrow 2)\right] \\
\epsilon_{\mathrm{comp}} & =-\left(\bar{\epsilon}_{2} \gamma^{M} \epsilon_{1}\right) \psi_{M}+\left[\xi_{2}^{N} \partial_{N} \epsilon_{1}+\frac{1}{4} \lambda_{2}^{A B} \gamma_{A B} \epsilon_{1}-(1 \leftrightarrow 2)\right]
\end{aligned}
$$

with $\gamma^{M}=\gamma^{A} e_{A}{ }^{M}$. The composite parameters depend explicitly on the fields of the 3D supergravity multiplet $\left(e_{M}^{A}, \psi_{M}, S\right)$, with $e_{A}^{M}$ being the inverse of $e_{M}^{A}$ and $\widehat{\omega}_{M A B}$ being the supercovariant spin connection (see $(3.4)$ ). The algebra is realized on the supergravity multiplet itself, as well as on other $3 \mathrm{D}$ multiplets such as the $3 \mathrm{D}$ scalar multiplet $\Phi_{3}(A)=$ $(A, \chi, F)$.

\subsection{Einstein boundary condition}

We are interested in constructing supersymmetric bulk-plus-boundary actions of the form

$$
S=\int_{\mathcal{M}} d^{3} x \mathcal{L}_{3}+\int_{\partial \mathcal{M}} d^{2} x \mathcal{L}_{2}
$$

\footnotetext{
${ }^{4}$ The gauge algebra of 4D $N=1$ sugra was first discussed in [27], and its closure if auxiliary fields are included was discussed in [2, 肘.

${ }^{5}$ Our conventions are: $M, N$ are curved 3D indices, $A, B$ are flat $3 \mathrm{D}$ indices, with decomposition $M=(m, 3)$ and $A=(a, \hat{3})$. The $3 \mathrm{D}$ gamma matrices satisfy $\gamma^{A} \gamma^{B}=\gamma^{A B}+\eta^{A B}$ with $\eta^{A B}=(-++)$ and $\gamma^{A} \gamma^{B} \gamma^{C}=$ $\gamma^{A B C}+\eta^{A B} \gamma^{C}+\eta^{B C} \gamma^{A}-\eta^{A C} \gamma^{B}$ with $\gamma^{A B C}=\varepsilon^{A B C}$. Our spinors are Majorana; $\bar{\psi}=\psi^{\mathrm{T}} C, C^{\mathrm{T}}=-C$, $C \gamma^{A} C^{-1}=-\left(\gamma^{A}\right)^{\mathrm{T}}$. Einstein transformations yield $\delta_{\xi} e_{M}{ }^{A}=\xi^{N} \partial_{N} e_{M}{ }^{A}+e_{N}{ }^{A} \partial_{M} \xi^{N}$, etc.; Lorentz and susy transformations are given in (3.6), (3.1) and 3.32.
} 
For notational simplicity, ${ }^{6}$ we choose the coordinates $x^{M}$ in such a way that the boundary $\partial \mathcal{M}$ is at $x^{3}=0$ and that $x^{3}>0$ in the bulk $\mathcal{M}$. The boundary has coordinates $x^{m}=\left(x^{0}, x^{1}\right)$. Under Einstein transformations, $\mathcal{L}_{3}$ is assumed to be a density, $\delta_{\xi} \mathcal{L}_{3}=\partial_{M}\left(\xi^{M} \mathcal{L}_{3}\right)$, so that

$$
\delta_{\xi} S=\int_{\partial \mathcal{M}} d^{2} x\left(-\xi^{3} \mathcal{L}_{3}+\delta_{\xi} \mathcal{L}_{2}\right)
$$

The standard way to achieve $\delta_{\xi} S=0$ is to impose a BC on the Einstein parameter,

$$
\xi^{3} \stackrel{\partial \mathcal{M}}{=} 0
$$

and take $\mathcal{L}_{2}$ to be a density under the induced Einstein transformations, $\delta_{\xi} \mathcal{L}_{2}=\partial_{m}\left(\xi^{m} \mathcal{L}_{2}\right)$. (We assume that the total $\partial_{m}$ derivative integrates to zero on the boundary.) In principle, one could investigate other ways to achieve $\delta_{\xi} S=0$ without imposing the $\mathrm{BC}$ (2.5). In this article, however, we will assume that this $\mathrm{BC}$ on the parameter $\xi^{M}$ has to be imposed.

\subsection{The unbroken half of bulk susy}

Consistency of the gauge algebra (2.1) with the BC (2.5) requires 11]

$$
\xi_{\text {comp }}^{3} \stackrel{\partial \mathcal{M}}{=} 0 \quad \Leftrightarrow \quad\left(\bar{\epsilon}_{2} \gamma^{A} \epsilon_{1}\right) e_{A} \stackrel{3}{\stackrel{\partial \mathcal{M}}{=}} 0
$$

It is convenient to choose a special Lorentz gauge, ${ }^{7}$

$$
e_{a}^{3}=0 \Rightarrow e_{m}^{\hat{3}}=0
$$

both on $\partial \mathcal{M}$ and in $\mathcal{M}$. (We shall later comment on the case when one does not impose this gauge.) As $e_{\hat{3}}^{3}$ is non-zero, the $\mathrm{BC}(2.6)$ now reduces to a field-independent requirement

$$
\bar{\epsilon}_{2} \gamma^{\hat{3}} \epsilon_{1} \stackrel{\partial \mathcal{M}}{=} 0
$$

Introducing projectors $P_{ \pm}=\frac{1}{2}\left(1 \pm \gamma^{\hat{3}}\right)$ and defining $\epsilon_{ \pm}=P_{ \pm} \epsilon$, we solve this BC by imposing (without loss of generality) the following $\mathrm{BC}$ on the susy parameter $\epsilon$,

$$
\epsilon_{-} \stackrel{\partial \mathcal{M}}{=} 0 \quad \Leftrightarrow \quad \epsilon \stackrel{\partial \mathcal{M}}{=} \epsilon_{+}
$$

The half of susy that is not broken by the boundary satisfies

$$
\epsilon_{+}=P_{+} \epsilon_{+}, \quad \bar{\epsilon}_{+}=\bar{\epsilon}_{+} P_{-}, \quad \gamma^{\hat{3}} \epsilon_{+}=\epsilon_{+}, \quad \bar{\epsilon}_{+}=-\bar{\epsilon}_{+} \gamma^{\hat{3}}
$$

\footnotetext{
${ }^{6}$ Our choice of coordinates $x^{M}$ does not impose an Einstein gauge as it does not restrict $\xi^{M}(x)$. It also does not imply that our boundary has to be flat, because it places no restrictions on (intrinsic or extrinsic) curvature.

${ }^{7}$ Note that the gauge $e_{a}{ }^{3}=e_{m}{ }^{\hat{3}}=0$ is opposite to the standard Kaluza-Klein choice [28], $e_{\hat{3}}{ }^{m}=e_{3}{ }^{a}=0$. It is the analog of the "time gauge" introduced by Schwinger [29] for the Hamiltonian analysis of gravity. (For the Hamiltonian analysis of the Dirac action in a curved space it was used by Kibble 30, and for the Hamiltonian formulation of $4 \mathrm{D} N=1$ supergravity it was used in 31]). In more mathematical terms, this gauge corresponds to the choice of a surface-compatible frame [32]. Its usefulness in the setting of supergravity on a manifold with boundary was emphasized in 19.
} 
The other half, parametrized by $\epsilon_{-}$, is broken by the boundary. It could, in principle, be restored by introducing appropriate Goldstone fields on the boundary, which would show that the breaking is spontaneous. However, in this article, we will only be interested in preserving the $\epsilon_{+}$susy.

\subsection{Modified $\epsilon_{+}$susy}

The gauge condition (2.7) is invariant under arbitrary $\xi^{m}$ and $\lambda^{a b}$ transformations, but not under $\lambda^{a \hat{3}}$ and $\epsilon_{+}$ones. Only a particular combination of $\lambda^{a \hat{3}}$ and $\epsilon_{+}$transformations survives in this gauge. We, therefore, introduce a modified $\epsilon_{+}$susy transformation,

$$
\delta_{Q}^{\prime}\left(\epsilon_{+}\right)=\delta_{Q}\left(\epsilon_{+}\right)+\delta_{L}\left(\lambda_{a \hat{3}}^{\prime}=-\bar{\epsilon}_{+} \psi_{a-}\right)
$$

which satisfies $\delta_{Q}^{\prime}\left(\epsilon_{+}\right) e_{m}^{\hat{3}}=0$. (We will use the notation $\delta_{\epsilon}^{\prime} \equiv \delta_{Q}^{\prime}\left(\epsilon_{+}\right)$.) It is this $\epsilon_{+}$susy transformation that we will use in the following constructions.

\subsection{The reduced gauge algebra}

We claim that the surviving gauge transformations, $\delta_{E}\left(\xi^{m}\right), \delta_{L}\left(\lambda^{a b}\right)$, and $\delta_{Q}^{\prime}\left(\epsilon_{+}\right)$, form a subalgebra of the 3D $N=1$ supergravity gauge algebra that is isomorphic to the (standard) 2D $N=(1,0)$ supergravity gauge algebra. The non-trivial part of the proof concerns the commutator of two (modified) $\epsilon_{+}$susy transformations. We find

$$
\left[\delta_{Q}^{\prime}\left(\epsilon_{1+}\right), \delta_{Q}^{\prime}\left(\epsilon_{2+}\right)\right]=\delta_{E}\left(\xi^{M}\right)+\delta_{L}\left(\lambda^{A B}\right)+\delta_{Q}(\epsilon)+\delta_{L}\left(\widetilde{\lambda}_{a \hat{3}}\right)
$$

where $^{8}$

$$
\begin{array}{ll}
\xi^{m}=2\left(\bar{\epsilon}_{2+} \gamma^{a} \epsilon_{1+}\right) e_{a}{ }^{m}, \quad \xi^{3}=0, \quad \epsilon=-\frac{1}{2} \xi^{n} \psi_{n}+\frac{1}{2} \xi^{n} \psi_{n-} \\
\lambda_{a b}=\xi^{n}\left[\widehat{\omega}_{n a b}-\frac{1}{2} \bar{\psi}_{a-} \gamma_{n} \psi_{b-}\right], \quad \lambda_{a \hat{3}}=\xi^{n}\left[\widehat{\omega}_{n a \hat{3}}+\frac{1}{2} S e_{n a}\right]
\end{array}
$$

The extra composite Lorentz transformation with

$$
\widetilde{\lambda}_{a \hat{3}}=-\bar{\epsilon}_{2+} \delta_{Q}^{\prime}\left(\epsilon_{1+}\right) \psi_{a-}-(1 \leftrightarrow 2)
$$

arises because the compensating Lorentz transformation in (2.11) is field-dependent. We see immediately that the (composite) $\epsilon_{-}$vanishes identically (without imposing $\psi_{m-}=0$ ), thanks to the contribution from the compensating Lorentz transformation. Using the results of the next section, one finds that [12]

$$
\lambda_{a \hat{3}}+\widetilde{\lambda}_{a \hat{3}}=\frac{1}{2} \xi^{n} \bar{\psi}_{n+} \psi_{a-}, \quad \widehat{\omega}_{n a b}-\frac{1}{2} \bar{\psi}_{a-} \gamma_{n} \psi_{b-}=\widehat{\omega}_{n a b}^{+}
$$

\footnotetext{
${ }^{8}$ The extra terms in $\lambda_{a b}$ and $\epsilon$ arise from the terms $\left(\lambda_{2}^{\prime}\right)_{a}{ }^{\hat{3}}\left(\lambda_{1}^{\prime}\right)_{\hat{3} b}$ and $\frac{1}{2} \lambda_{2}^{\prime a \hat{3}} \gamma_{a \hat{3}} \epsilon_{1+}$ in 2.2 upon using the Fierz identities $\left(\bar{\epsilon}_{+} \psi_{-}\right)\left(\bar{\phi}_{-} \eta_{+}\right)=-\frac{1}{2}\left(\bar{\epsilon}_{+} \gamma^{c} \eta_{+}\right)\left(\bar{\phi}_{-} \gamma_{c} \psi_{-}\right)$and $\left(\bar{\epsilon}_{+} \psi_{-}\right) \phi_{-}=-\left(\bar{\epsilon}_{+} \phi_{-}\right) \psi_{-}$.
} 
where $\widehat{\omega}_{n a b}^{+}$is the standard supercovariant connection constructed out of $e_{m}{ }^{a}$ and $\psi_{m+}$. This brings (2.12) to the form

$$
\left[\delta_{Q}^{\prime}\left(\epsilon_{1+}\right), \delta_{Q}^{\prime}\left(\epsilon_{2+}\right)\right]=\delta_{E}\left(\xi^{m}\right)+\delta_{L}\left(\lambda_{a b}=\xi^{n} \widehat{\omega}_{n a b}^{+}\right)+\delta_{Q}^{\prime}\left(\epsilon_{+}=-\frac{1}{2} \xi^{n} \psi_{n+}\right)
$$

which is the standard form of the $2 \mathrm{D} N=(1,0)$ (local) susy algebra. We emphasize that we have identified this subalgebra without imposing any boundary conditions on supergravity fields. Accordingly, this identification works for any hypersurface $x^{3}=$ const parallel to the boundary $\partial \mathcal{M}$.

\section{Co-dimension one submultiplets}

Having proved that the $3 \mathrm{D} N=1$ supergravity gauge algebra reduces to the $2 \mathrm{D} N=(1,0)$ supergravity gauge algebra on the hypersurfaces parallel to the boundary, we are guaranteed that the 3D multiplets can be decomposed into a set of 2D submultiplets. In this section, we will describe these submultiplets for the 3D supergravity and the 3D scalar multiplets.

\subsection{D supergravity multiplet}

The 3D supergravity multiplet, $\left(e_{M}^{A}, \psi_{M}, S\right)$, enjoys the following susy transformations,

$$
\delta_{\epsilon} e_{M}^{A}=\bar{\epsilon} \gamma^{A} \psi_{M}, \quad \delta_{\epsilon} \psi_{M}=2 \widehat{D}_{M} \epsilon, \quad \delta_{\epsilon} S=\frac{1}{2} \bar{\epsilon} \gamma^{M N} \widehat{\psi}_{M N}
$$

where $\widehat{\psi}_{M N}=\widehat{D}_{M} \psi_{N}-\widehat{D}_{N} \psi_{M}$ is the supercovariant gravitino field strength and

$$
\widehat{D}_{M} \epsilon=D_{M}(\widehat{\omega}) \epsilon+\frac{1}{4} \gamma_{M} \epsilon S, \quad \widehat{D}_{M} \psi_{N}=D_{M}(\widehat{\omega}) \psi_{N}-\frac{1}{4} \gamma_{N} \psi_{M} S
$$

The covariant derivatives $D_{M}$ are only Lorentz covariant, so that

$$
D_{M}(\widehat{\omega}) \psi_{N}=\partial_{M} \psi_{N}+\frac{1}{4} \widehat{\omega}_{M A B} \gamma^{A B} \psi_{N}
$$

and the supercovariant spin connection is given by

$$
\begin{array}{r}
\widehat{\omega}_{M A B}=\omega(e)_{M A B}+\kappa_{M A B}, \quad \kappa_{M A B}=\frac{1}{4}\left(\bar{\psi}_{M} \gamma_{A} \psi_{B}-\bar{\psi}_{M} \gamma_{B} \psi_{A}+\bar{\psi}_{A} \gamma_{M} \psi_{B}\right) \\
\omega(e)_{M A B}=\frac{1}{2}\left(C_{M A B}-C_{M B A}-C_{A B M}\right), \quad C_{M N}{ }^{A}=\partial_{M} e_{N}{ }^{A}-\partial_{N} e_{M}{ }^{A}
\end{array}
$$

where we use the standard conversion of indices, $\psi_{A}=e_{A}{ }^{M} \psi_{M}$, etc. The supercovariant spin connection has the following susy transformation,

$$
\delta_{\epsilon} \widehat{\omega}_{M A B}=\frac{1}{2} \bar{\epsilon}\left(\gamma_{B} \widehat{\psi}_{M A}-\gamma_{A} \widehat{\psi}_{M B}-\gamma_{M} \widehat{\psi}_{A B}\right)-\frac{1}{2}\left(\bar{\epsilon} \gamma_{A B} \psi_{M}\right) S
$$

Under a 3D Lorentz transformation, we have

$$
\delta_{\lambda} e_{M}^{A}=\lambda^{A B} e_{M B}, \quad \delta_{\lambda} \psi_{M}=\frac{1}{4} \lambda^{A B} \gamma_{A B} \psi_{M}, \quad \delta_{\lambda} S=0, \quad \delta_{\lambda} \widehat{\omega}_{M A B}=-D(\widehat{\omega})_{M} \lambda_{A B}
$$

These Lorentz transformations will play a role as the (modified) $\epsilon_{+}$susy transformation (2.11) involves a compensating Lorentz transformation. 


\subsection{Co-dimension one split}

To identify co-dimension one submultiplets of the supergravity multiplet, we first split the indices, $M=(m, 3), A=(a, \hat{3})$, and the spinors, $\epsilon=\epsilon_{+}+\epsilon_{-}$. The resulting component fields (and parameters) can be formally assigned parities (in a way consistent with the susy transformations) as follows,

$$
\begin{aligned}
& \text { even: } e_{m}{ }^{a} e_{3}{ }^{\hat{3}} \quad \omega_{m a b} \quad \omega_{3 a \hat{3}} \quad \psi_{m+} \psi_{3-} \epsilon_{+} \quad \partial_{m} \\
& \text { odd: } e_{3}{ }^{a} \quad e_{m}{ }^{\hat{3}}=0 \quad \omega_{3 a b} \quad \omega_{m a \hat{3}} \quad S \quad \psi_{m-} \quad \psi_{3+} \quad \epsilon_{-}=0 \quad \partial_{3}
\end{aligned}
$$

(The vanishing of $e_{m}{ }^{\hat{3}}$ and $\epsilon_{-}$correspond to our Lorentz gauge choice (2.7) and the restriction (2.9) on susy, respectively.) Co-dimension one multiplets will have definite parities as well.

In general, the induced metric on the $x^{3}=$ const slices is $g_{m n}=e_{m}{ }^{a} e_{n a}+e_{m}{ }^{\hat{3}} e_{n \hat{3}}$. With our choice of the Lorentz gauge, however, we have $g_{m n}=e_{m}{ }^{a} e_{n a}$, so that $e_{m}{ }^{a}$ is the induced vielbein. One can also easily check that $\omega(e)_{m a b}$ coincides with the torsion-free spin connection constructed out of $e_{m}{ }^{a}$, whereas $\omega(e)_{m a \hat{3}} e_{n}{ }^{a}$ coincides, up to a convention-dependent sign, with the extrinsic curvature tensor [19]. We fix the sign by defining 9

$$
K_{m n}=\omega(e)_{m a \hat{3}} e_{n}^{a}
$$

In our gauge, $e_{m}{ }^{\hat{3}}=e_{a}{ }^{3}=0$, we have $e_{m}{ }^{a} e_{a}{ }^{n}=\delta_{m}{ }^{n}, e_{a}{ }^{m} e_{m}{ }^{b}=\delta_{a}{ }^{b}$ and $e_{3}{ }^{\hat{3}} e_{\hat{3}}{ }^{3}=1$, as well as

$$
\begin{aligned}
& \gamma_{m}=e_{m}{ }^{a} \gamma_{a}, \quad \gamma_{3}=e_{3}{ }^{a} \gamma_{a}+e_{3}^{\hat{3}} \gamma_{\hat{3}}, \quad \gamma^{m}=\gamma^{a} e_{a}{ }^{m}+\gamma^{\hat{3}} e_{\hat{3}}^{m}, \quad \gamma^{3}=\gamma^{\hat{3}} e_{\hat{3}}^{3} \\
& \psi_{a}=e_{a}{ }^{m} \psi_{m}, \quad \psi_{\hat{3}}=e_{\hat{3}}{ }^{m} \psi_{m}+e_{\hat{3}}{ }^{3} \psi_{3}
\end{aligned}
$$

We will also use $K_{m a}=\omega(e)_{m a \hat{3}}$ and $K_{b a}=e_{b}{ }^{m} K_{m a}$. Noting that $\widehat{\omega}_{m a \hat{3}}$ is not supercovariant under the (modified) $\epsilon_{+}$susy, we define the supercovariant extrinsic curvature tensor as

$$
\widehat{K}_{m a}=\widehat{\omega}_{m a \hat{3}}-\frac{1}{2} \bar{\psi}_{m+} \psi_{a-}
$$

Using $\bar{\psi}_{m} \psi_{a}=\bar{\psi}_{m+} \psi_{a-}+\bar{\psi}_{m-} \psi_{a+}$ and $\bar{\psi}_{m} \gamma_{\hat{3}} \psi_{a}=-\bar{\psi}_{m+} \psi_{a-}+\bar{\psi}_{m-} \psi_{a+}$, we find that

$$
\widehat{K}_{m a}=K_{m a}+\frac{1}{4}\left(\bar{\psi}_{m} \gamma_{a} \psi_{\hat{3}}-\bar{\psi}_{m} \psi_{a}+\bar{\psi}_{a} \gamma_{m} \psi_{\hat{3}}\right)
$$

As the bosonic extrinsic curvature tensor is symmetric, $K_{a b}=K_{b a}$, the supercovariant extrinsic curvature tensor is symmetric as well, $\widehat{K}_{a b}=\widehat{K}_{b a}$.

\footnotetext{
${ }^{9}$ The extrinsic curvature is usually defined by $K_{M N}= \pm P_{M}{ }^{K} P_{N}{ }^{L} \nabla_{K} n_{L}$ where $P_{M}{ }^{K}=\delta_{M}{ }^{K}-n_{M} n^{K}$ and $\nabla_{K} n_{L}=\partial_{K} n_{L}-\Gamma_{K L}{ }^{S} n_{S}$. In our gauge and with our choice of coordinates, $n_{M}=\left(0,0,-e_{3}^{\hat{3}^{\hat{3}}}\right)$ and $K_{m n}=\mp \Gamma_{m n}{ }^{3} n_{3}= \pm \Gamma_{m n}{ }^{3} e_{3}{ }^{\hat{3}}$. The vielbein postulate yields $\Gamma_{m n}{ }^{3} e_{3}{ }^{\hat{3}}=-\omega_{m a}{ }^{\hat{3}} e_{n}{ }^{a}$. (See appendices in 11] and 19] for more details and references.) Our sign choice is then $K_{M N}=-P_{M}{ }^{K} P_{N}{ }^{L} \nabla_{K} n_{L}$.
} 


\subsection{Induced supergravity multiplet}

Under the (modified) $\epsilon_{+}$susy (2.11), the induced vielbein transforms as follows,

$$
\delta_{\epsilon}^{\prime} e_{m}^{a}=\bar{\epsilon}_{+} \gamma^{a} \psi_{m+}
$$

(The compensating Lorentz transformation does not contribute here as $\lambda^{\prime a \hat{3}} e_{m \hat{3}}$ vanishes in our gauge.) The variation of $\psi_{m+}$ gives

$$
\delta_{\epsilon}^{\prime} \psi_{m+}=2\left(\partial_{m}+\frac{1}{4} \widehat{\omega}_{m a b} \gamma^{a b}\right) \epsilon_{+}+\frac{1}{2} \lambda_{a \hat{3}}^{\prime} \gamma^{a \hat{3}} \psi_{m-}
$$

where $\lambda_{a \hat{3}}^{\prime}=-\bar{\epsilon}_{+} \psi_{a-}$. Performing the following decomposition,

$$
\begin{aligned}
& \widehat{\omega}_{m a b}=\widehat{\omega}_{m a b}^{+}+\kappa_{m a b}^{-}, \quad \kappa_{m a b}^{-}=\frac{1}{4}\left(\bar{\psi}_{m-} \gamma_{a} \psi_{b-}-\bar{\psi}_{m-} \gamma_{b} \psi_{a-}+\bar{\psi}_{a-} \gamma_{m} \psi_{b-}\right) \\
& \widehat{\omega}_{m a b}^{+}=\omega(e)_{m a b}+\kappa_{m a b}^{+}, \quad \kappa_{m a b}^{+}=\frac{1}{4}\left(\bar{\psi}_{m+} \gamma_{a} \psi_{b+}-\bar{\psi}_{m+} \gamma_{b} \psi_{a+}+\bar{\psi}_{a+} \gamma_{m} \psi_{b+}\right)
\end{aligned}
$$

we observe that $\widehat{\omega}_{m a b}^{+}$is the (standard) supercovariant spin connection for the $2 \mathrm{D}$ (induced) vielbein $e_{m}{ }^{a}$. Defining the 2D (Lorentz) covariant derivative as

$$
D_{m}^{\prime}\left(\widehat{\omega}^{+}\right) \epsilon=\partial_{m} \epsilon+\frac{1}{4} \widehat{\omega}_{m a b}^{+} \gamma^{a b} \epsilon
$$

we arrive at

$$
\delta_{\epsilon}^{\prime} \psi_{m+}=2 D_{m}^{\prime}\left(\widehat{\omega}^{+}\right) \epsilon_{+}+\frac{1}{2} \kappa_{m a b}^{-} \gamma^{a b} \epsilon_{+}+\frac{1}{2} \lambda_{a \hat{3}}^{\prime} \gamma^{a \hat{3}} \psi_{m-}
$$

We claim that the last two terms cancel each other. To prove this, we first observe that the antisymmetrization in any three $2 \mathrm{D}$ vector indices gives zero, $[a b c]=0$, which yields

$$
\kappa_{m a b}^{-}=\frac{1}{2} \bar{\psi}_{a-} \gamma_{m} \psi_{b-}
$$

Second, the identity $\gamma^{a b}=\epsilon^{a b \hat{3}} \gamma_{\hat{3}}$ accounts for a useful trick,

$$
\gamma^{a b} \epsilon_{+}\left(\bar{\psi}_{a-} \gamma_{m} \psi_{b-}\right)=-\epsilon_{+}\left(\bar{\psi}_{a-} \gamma_{m} \gamma^{a b} \psi_{b-}\right)
$$

Finally, gamma-matrix algebra reduces the last term to $2 \epsilon_{+}\left(\bar{\psi}_{a-} \gamma^{a} \psi_{m-}\right)$ and the Fierz transformation gives

$$
\gamma^{a b} \epsilon_{+}\left(\bar{\psi}_{a-} \gamma_{m} \psi_{b-}\right)=-2 \gamma^{a} \psi_{m-}\left(\bar{\epsilon}_{+} \psi_{a-}\right)
$$

which proves our statement and gives us the final result,

$$
\delta_{\epsilon}^{\prime} e_{m}^{a}=\bar{\epsilon}_{+} \gamma^{a} \psi_{m+}, \quad \delta_{\epsilon}^{\prime} \psi_{m+}=2 D_{m}^{\prime}\left(\widehat{\omega}^{+}\right) \epsilon_{+}
$$

This shows that $\left(e_{m}{ }^{a}, \psi_{m+}\right)$ is the (standard) $2 \mathrm{D} N=(1,0)$ supergravity multiplet. 


\subsection{Radion multiplet}

In order to identify further submultiplets, we recall the basics of the $2 \mathrm{~d} N=(1,0)$ supergravity tensor calculus [25]. Besides the supergravity multiplet we have just identified, there are two other basic multiplets, the scalar multiplet $\Phi_{2}(A)=\left(A, \zeta_{-}\right)$and the spinor multiplet $\Psi_{2}\left(\zeta_{+}\right)=\left(\zeta_{+}, F\right)$. They transform by definition as follows,

$$
\begin{array}{ll}
\delta_{\epsilon}^{\prime} A=\bar{\epsilon}_{+} \zeta_{-}, & \delta_{\epsilon}^{\prime} \zeta_{-}=\gamma^{a} \epsilon_{+} \widehat{D}_{a}^{\prime} A \\
\delta_{\epsilon}^{\prime} \zeta_{+}=F \epsilon_{+}, & \delta_{\epsilon}^{\prime} F=\bar{\epsilon}_{+} \gamma^{a} \widehat{D}_{a}^{\prime} \zeta_{+}
\end{array}
$$

where $\widehat{D}_{a}^{\prime} A=\partial_{a} A-\frac{1}{2} \bar{\psi}_{a+} \chi_{-}$and $\widehat{D}_{a}^{\prime} \zeta_{+}=D_{a}^{\prime}\left(\widehat{\omega}^{+}\right) \zeta_{+}-\frac{1}{2} F \psi_{a+}$ are supercovariant derivatives.

With these definitions, we now claim that

$$
\Phi_{2}\left(e_{3}{ }^{\hat{3}}\right)=\left(e_{3}^{\hat{3}}, \quad-e_{3}{ }^{\hat{3}} \psi_{\hat{3}-}\right)
$$

is a good $2 \mathrm{D} N=(1,0)$ scalar multiplet which we will call the radion multiplet. ${ }^{10}$ First of all, we observe that $e_{3}{ }^{\hat{3}}$ is indeed a scalar under the $\xi^{m}$ and $\lambda^{a b}$ transformations. The non-trivial part in this statement is that in

$$
\delta_{\xi} e_{3}^{\hat{3}}=\xi^{n} \partial_{n} e_{3}^{\hat{3}}+e_{n}{ }^{\hat{3}} \partial_{3} \xi^{n}
$$

the last term vanishes in our gauge. Next, we apply the (modified) $\epsilon_{+}$susy to $e_{3}{ }^{\hat{3}}$ and find

$$
\delta_{\epsilon}^{\prime} e_{3}^{\hat{3}}=\bar{\epsilon}_{+} \gamma^{\hat{3}} \psi_{3}+\lambda^{\prime \hat{3} a} e_{3 a}=\bar{\epsilon}_{+}\left(-\psi_{3-}+e_{3}{ }^{a} \psi_{a-}\right)=\bar{\epsilon}_{+}\left(-e_{3}{ }^{\hat{3}} \psi_{\hat{3}-}\right)
$$

which identifies the superpartner of $e_{3}{ }^{\hat{3}}$ as $\zeta_{-}=-e_{3}{ }^{\hat{3}} \psi_{\hat{3}-}$. To check that the variation of $\zeta_{-}$ has the correct form is a bit more involved. The details will be presented in [12]. The key intermediate statement is

$$
\delta_{\epsilon}^{\prime} \psi_{\hat{3}-}=P_{-}\left[e_{\hat{3}}^{M} \delta \psi_{M}+\psi_{M} \delta e_{\hat{3}}^{M}\right]=\gamma^{a} \epsilon_{+}\left[\widehat{\omega}_{\hat{3} a \hat{3}}-\frac{1}{2} \bar{\psi}_{\hat{3}+} \psi_{a-}\right]
$$

Next, in our gauge, it is easy to prove that

$$
\widehat{\omega}_{\hat{3} a \hat{3}}=-e_{\hat{3}}^{3} \partial_{a} e_{3 \hat{3}}+\frac{1}{2}\left(\bar{\psi}_{\hat{3}+} \psi_{a-}-\bar{\psi}_{\hat{3}-} \psi_{a+}\right)
$$

Finally, the contribution $\psi_{\hat{3}-} \delta e_{3}{ }^{\hat{3}}$ vanishes thanks to the identity $\left(\bar{\epsilon}_{+} \psi_{-}\right) \psi_{-}=0$. Collecting the pieces, we find that $\delta \zeta_{-}$has the required form, which proves that $(3.22)$ is a good $2 \mathrm{D}$ $N=(1,0)$ scalar multiplet.

\footnotetext{
${ }^{10}$ The term "radion" refers to a field parametrizing the radius of the extra dimension 33]. In our case, proper distances in the $x^{3}$ direction must be measured with $g_{33}=e_{3}{ }^{\hat{3}} e_{3 \hat{3}}+e_{3}{ }^{a} e_{3 a}$, which is not given by $e_{3}{ }^{\hat{3}}$ alone. Nonetheless, we will call $\Phi\left(e_{3}{ }^{\hat{3}}\right)$ the radion multiplet.
} 


\subsection{Extrinsic curvature multiplet}

So far, we have found two even submultiplets, the induced supergravity and the radion multiplets. Now we will present an important odd submultiplet, the extrinsic curvature (scalar) multiplet. The starting point is the (modified) $\epsilon_{+}$susy transformation of $\psi_{m-}$,

$$
\delta_{\epsilon}^{\prime} \psi_{m-}=\widehat{\omega}_{m a \hat{3}} \gamma^{a \hat{3}} \epsilon_{+}+\frac{1}{2} \gamma_{m} \epsilon_{+} S+\frac{1}{2} \lambda_{a \hat{3}}^{\prime} \gamma^{a \hat{3}} \psi_{m+}
$$

Observing that $\delta_{\epsilon}^{\prime} e_{a}^{m}=-\left(\bar{\epsilon}_{+} \gamma^{b} \psi_{a+}\right) e_{b}{ }^{m}$, we find, after some Fierzing,

$$
\delta_{\epsilon}^{\prime} \psi_{a-}=\gamma^{b} \epsilon_{+}\left[\widehat{K}_{a b}+\frac{1}{2} \eta_{a b} S\right]
$$

where $\widehat{K}_{a b}$ is the (symmetric) supercovariant extrinsic curvature tensor defined in (3.10). Contracting this expression with $\gamma^{a}$, we find

$$
\delta_{\epsilon}^{\prime}\left(\gamma^{a} \psi_{a-}\right)=(\widehat{K}+S) \epsilon_{+}
$$

where $\widehat{K}=\eta^{a b} \widehat{K}_{a b}$ is the (supercovariant) extrinsic curvature scalar. Noting that $\gamma^{a} \psi_{a-}$ behaves as $\zeta_{+}$, we claim that

$$
\Psi_{2}\left(\gamma^{a} \psi_{a-}\right)=\left(\gamma^{a} \psi_{a-}, \quad \widehat{K}+S\right)
$$

is a good 2D $N=(1,0)$ spinor multiplet. The proof consists in demonstrating that

$$
\delta_{\epsilon}^{\prime}(\widehat{K}+S)=\bar{\epsilon}_{+} \gamma^{a} D_{a}^{\prime}\left(\widehat{\omega}^{+}\right)\left[\gamma^{b} \psi_{b-}\right]-\frac{1}{2}(\widehat{K}+S)\left(\bar{\epsilon}_{+} \gamma^{a} \psi_{a+}\right)
$$

The details of the proof will be presented in [12], where we will also discuss an extrinsic curvature tensor multiplet as well as a submultiplet that starts with $e_{3}{ }^{a}$.

\subsection{Submultiplets of the 3D scalar multiplet}

In 3D $N=1$ supergravity, there is only one type of matter multiplet, the scalar multiplet $\Phi_{3}(A)=(A, \chi, F)$. (Other multiplets can be constructed by adding extra Lorentz indices.) The susy transformations of this multiplet are

$$
\delta_{\epsilon} A=\bar{\epsilon} \chi, \quad \delta_{\epsilon} \chi=\gamma^{M} \epsilon \widehat{D}_{M} A+F \epsilon, \quad \delta_{\epsilon} F=\bar{\epsilon} \gamma^{M} \widehat{D}_{M} \chi-\frac{1}{4} S \bar{\epsilon} \chi
$$

where $\widehat{D}_{M} A=\partial_{M} A-\frac{1}{2} \bar{\psi}_{M} \chi$ and $\widehat{D}_{M} \chi=D_{M}(\widehat{\omega}) \chi-\frac{1}{2} \gamma^{N} \psi_{M} \widehat{D}_{N} A-\frac{1}{2} F \psi_{M}$ are supercovariant derivatives. Under the (modified) $\epsilon_{+}$susy, this $3 \mathrm{D}$ multiplet splits into the following two $2 \mathrm{D}$ $N=(1,0)$ submultiplets, ${ }^{11}$

$$
\Phi_{2}(A)=\left(A, \chi_{-}\right), \quad \Psi_{2}\left(\chi_{+}\right)=\left(\chi_{+}, \quad F+\widehat{D}_{\hat{3}} A-\frac{1}{2} \bar{\psi}_{a-} \gamma^{a} \chi_{-}\right)
$$

\footnotetext{
${ }^{11}$ We note that our co-dimension one multiplets contain terms of the type "odd · odd" that are set to zero in the approach of [20, 21]. For example, let us take $F$ to be even, so that $\chi_{+}$is even and $\chi_{-}$is odd. The multiplet $\Psi_{2}\left(\chi_{+}\right)$is then even and contains an explicit product of odd fields, $\bar{\psi}_{a-} \gamma^{a} \chi_{-}$. Such a product is also present in the radion multiplet 3.22 via the term $e_{3}{ }^{a} \psi_{a-}$ inside $\zeta_{-}=-e_{3}{ }^{\hat{3}} \psi_{\hat{3}_{-}}$. For dimensions higher than $3 \mathrm{D}$, such products also appear in the induced supergravity multiplet 12 ].
} 
The proof consists in showing that

$$
\begin{aligned}
\delta_{\epsilon}^{\prime} A & =\bar{\epsilon}_{+} \chi_{-}, \quad \delta_{\epsilon}^{\prime} \chi_{-}=\gamma^{a} \epsilon_{+} \widehat{D}_{a}^{\prime} A \\
\delta_{\epsilon}^{\prime} \chi_{+} & =F_{2} \epsilon_{+}, \quad F_{2} \equiv F+\widehat{D}_{\hat{3}} A-\frac{1}{2} \bar{\psi}_{a-} \gamma^{a} \chi_{-} \\
\delta_{\epsilon}^{\prime} F_{2} & =\bar{\epsilon}_{+} \gamma^{a} D^{\prime}\left(\widehat{\omega}^{+}\right)_{a} \chi_{+}-\frac{1}{2}\left(\bar{\epsilon}_{+} \gamma^{a} \psi_{a+}\right) F_{2}
\end{aligned}
$$

where $\widehat{D}_{a}^{\prime} A=e_{a}^{m}\left(\partial_{m} A-\frac{1}{2} \bar{\psi}_{m+} \chi_{-}\right)$and $\widehat{D}_{\hat{3}} A=e_{\hat{3}}{ }^{M}\left(\partial_{M} A-\frac{1}{2} \bar{\psi}_{M} \chi\right)$. The proof is straightforward, except for the $\delta_{\epsilon}^{\prime} F_{2}$ part that we will discuss in [12].

\subsection{Separately susy boundary actions}

In the 2D $N=(1,0)$ supergravity tensor calculus [25], susy actions are constructed from spinor multiplets $\Psi_{2}\left(\zeta_{+}\right)=\left(\zeta_{+}, F\right)$ with the help of the following $F$-density formula,

$$
\mathcal{L}_{F}\left[\Psi_{2}\left(\zeta_{+}\right)\right]=e_{2}\left[F+\frac{1}{2} \bar{\psi}_{a+} \gamma^{a} \zeta_{+}\right]
$$

where $e_{2}=\operatorname{det} e_{m}{ }^{a}$. In our case, this formula can be directly applied to constructing (separately) susy invariant boundary actions. Indeed, under the (modified) $\epsilon_{+}$susy, we have

$$
\delta_{\epsilon}^{\prime} \mathcal{L}_{F}\left[\Psi_{2}\left(\zeta_{+}\right)\right]=\partial_{m}\left[e_{2}\left(\bar{\epsilon}_{+} \gamma^{a} \zeta_{+}\right) e_{a}^{m}\right]
$$

and the total $\partial_{m}$ derivative integrates to zero on the boundary. Therefore,

$$
\int_{\partial \mathcal{M}} d^{2} x e_{2}\left[F+\frac{1}{2} \bar{\psi}_{a+} \gamma^{a} \zeta_{+}\right]
$$

is a (separately) susy boundary action for a general spinor multiplet $\Psi_{2}\left(\zeta_{+}\right)=\left(\zeta_{+}, F\right)$. For example, we can apply this formula to the extrinsic curvature multiplet (3.30) to obtain

$$
\int_{\partial \mathcal{M}} d^{2} x e_{2}\left[\widehat{K}+S+\frac{1}{2} \bar{\psi}_{a+} \gamma^{a} \gamma^{b} \psi_{b-}\right]
$$

which is (separately) supersymmetric under the (modified) $\epsilon_{+}$susy (2.11).

\section{Susy bulk-plus-boundary actions}

In this section, we will find an extension of the 3D F-density formula that makes it very easy to construct susy bulk-plus-boundary actions. We will then show how this formula can be written in terms of co-dimension one submultiplets. Finally, we will use it to supersymmetrize the York-Gibbons-Hawking construction. 


\subsection{The " $F+A$ " formula}

In the 3D $N=1$ supergravity tensor calculus [24], susy actions are constructed from scalar multiplets $\Phi_{3}(A)=(A, \chi, F)$ using the following $F$-density formula,

$$
\mathcal{L}_{F}\left[\Phi_{3}(A)\right]=e_{3}\left[F+\frac{1}{2} \bar{\psi}_{M} \gamma^{M} \chi+\frac{1}{4} A \bar{\psi}_{M} \gamma^{M N} \psi_{N}+A S\right]
$$

where $e_{3}=\operatorname{det} e_{M}{ }^{A}$. Under 3D susy, this density transforms into a total 3D derivative,

$$
\delta_{\epsilon} \mathcal{L}_{F}\left[\Phi_{3}(A)\right]=\partial_{M}\left[e_{3}\left(\bar{\epsilon} \gamma^{M} \chi+A \bar{\epsilon} \gamma^{M N} \psi_{N}\right)\right]
$$

In the presence of a boundary, the bulk $F$-density does not give rise to a separately susy bulk action because the total derivative yields a boundary term,

$$
\int_{\mathcal{M}} d^{3} x \delta_{\epsilon} \mathcal{L}_{F}\left[\Phi_{3}(A)\right]=-\int_{\partial \mathcal{M}} d^{2} x e_{2}\left(\bar{\epsilon} \gamma^{\hat{3}} \chi+A \bar{\epsilon} \gamma^{\hat{3} a} \psi_{a}\right)
$$

We used that, in our gauge, $e_{a}^{3}=0$ and $e_{3} e_{3}^{3}=e_{2}$. Noting that $\mathcal{L}_{F}\left[\Phi_{3}(A)\right]$ is a Lorentz scalar, the (modified) $\epsilon_{+}$susy transformation (2.11) gives

$$
\int_{\mathcal{M}} d^{3} x \delta_{\epsilon}^{\prime} \mathcal{L}_{F}\left[\Phi_{3}(A)\right]=\int_{\partial \mathcal{M}} d^{2} x e_{2}\left(\bar{\epsilon}_{+} \chi_{-}+A \bar{\epsilon}_{+} \gamma^{a} \psi_{a+}\right)
$$

Noting that $\delta_{\epsilon}^{\prime} A=\bar{\epsilon}_{+} \chi_{-}$and $\delta_{\epsilon}^{\prime} e_{2}=e_{2}\left(\bar{\epsilon}_{+} \gamma^{a} \psi_{a+}\right)$, we can construct a boundary action whose variation cancels (4.4). The following bulk-plus-boundary action,

$$
S_{F+A}=\int_{\mathcal{M}} d^{3} x \mathcal{L}_{F}\left[\Phi_{3}(A)\right]-\int_{\partial \mathcal{M}} d^{2} x e_{2} A
$$

is invariant under the (modified) $\epsilon_{+}$susy. We call this the " $F+A$ " formula. ${ }^{12}$

\subsection{Extended $F$-density}

As we will demonstrate explicitly in [12], the boundary $A$-term can also be written as a bulk contribution thanks to the following relation,

$$
-\int_{\partial \mathcal{M}} d^{2} x e_{2} A=\int_{\mathcal{M}} d^{3} x e_{3}\left(\partial_{\hat{3}} A+K A\right)
$$

This allows us to define an extended $F$-density

$$
\mathcal{L}_{F}^{\prime}\left[\Phi_{3}(A)\right]=\mathcal{L}_{F}\left[\Phi_{3}(A)\right]+e_{3}\left(\partial_{\hat{3}} A+K A\right)
$$

\footnotetext{
${ }^{12}$ The " $F+A$ " formula (4.5) has a natural extension to the case when the Lorentz gauge (2.7) is not imposed [12]. We only have to replace $e_{2}=\operatorname{det}\left(e_{m}{ }^{a}\right)$ with the determinant of the induced vielbein $e_{2}^{\prime}=\operatorname{det}\left(e_{m}^{\prime}{ }^{a}\right)$ which satisfies $e_{m}^{\prime}{ }^{a} e_{n a}^{\prime}=e_{m}{ }^{a} e_{n a}+e_{m}{ }^{\hat{3}} e_{n \hat{3}}$. The resulting bulk-plus-boundary action is susy under the half of bulk susy defined by $\gamma^{3} \epsilon_{+}=\sqrt{g^{33}} \epsilon_{+}$. Note that this makes the susy parameter $\epsilon_{+}$field-dependent which makes the analysis of the gauge algebra more subtle [12].
} 
whose integral over the bulk $\mathcal{M}$ reproduces the bulk-plus-boundary " $F+A$ " formula (4.5). Under the (modified) $\epsilon_{+}$susy, this extended 3D F-density behaves like the ordinary 2D Fdensity (that is, it varies into a total $\partial_{m}$ derivative). Therefore, we expect that it should be possible to rewrite it as a $2 \mathrm{D} F$-density of some $2 \mathrm{D} N=(1,0)$ spinor multiplet, ${ }^{13}$

$$
\mathcal{L}_{F}^{\prime}\left[\Phi_{3}(A)\right]=\mathcal{L}_{F}\left[\Psi_{2}\left(\zeta_{+}\right)\right]
$$

This is indeed possible, and we find [12]

$$
\Psi_{2}\left(\zeta_{+}\right)=\Phi_{2}\left(e_{3}^{\hat{3}}\right) \times\left[\Psi_{2}\left(\chi_{+}\right)+\Psi_{2}\left(\gamma^{a} \psi_{a-}\right) \times \Phi_{2}(A)\right]
$$

where $\Phi_{2}(A)$ and $\Psi_{2}\left(\chi_{+}\right)$are the submultiplets (3.33) of the 3D scalar multiplet $\Phi_{3}(A)$, whereas $\Phi_{2}\left(e_{3}{ }^{\hat{3}}\right)$ and $\Psi_{2}\left(\gamma^{a} \psi_{a-}\right)$ are the radion and the extrinsic curvature multiplets, respectively. To derive this result, one needs the multiplication formula

$$
\left(A, \zeta_{-}\right) \times\left(\zeta_{+}, F\right)=\left(A \zeta_{+}, \quad A F-\bar{\zeta}_{-} \zeta_{+}\right)
$$

which is part of the $2 \mathrm{D} N=(1,0)$ tensor calculus [25].

\subsection{Super-York-Gibbons-Hawking construction}

The " $F+A$ " formula (4.5) can be applied, in particular, to the 3D scalar curvature multiplet, ${ }^{14}$

$$
\Phi_{3}(S)=\left(S, \quad \frac{1}{2} \gamma^{M N} \psi_{M N}-\frac{1}{2} \gamma^{M} \psi_{M} S, \quad \frac{1}{2} R(\widehat{\omega})-\frac{1}{2} \bar{\psi}^{M} \gamma^{N} \psi_{M N}+\frac{1}{4} S \bar{\psi}^{M} \psi_{M}-\frac{3}{4} S^{2}\right)
$$

We immediately obtain the following bulk-plus-boundary action,

$$
S_{S G}=\int_{\mathcal{M}} d^{3} x e_{3}\left[\frac{1}{2} R(\widehat{\omega})+\frac{1}{2} \bar{\psi}_{M} \gamma^{M N K} D(\widehat{\omega})_{N} \psi_{K}+\frac{1}{4} S^{2}\right]-\int_{\partial \mathcal{M}} d^{2} x e_{2} S
$$

which is, by construction, invariant under the (modified) $\epsilon_{+}$susy (without using any boundary conditions). However, when one tries to apply the variational principle to this action, one runs into a problem because the bulk auxiliary field $S$ appears linearly on the boundary. (Its field equation would require $e_{2}$ to vanish, which is too strong.) This can be cured by adding a

\footnotetext{
${ }^{13}$ In the superfield language, this corresponds to giving a prescription for writing 3D locally susy actions in terms of $2 \mathrm{D}$ superfields. For rigid susy, similar constructions are known in various dimensions [14. For the linearized 5D supergravity, the description in terms of 4D superfields was given in 34. For the full non-linear $5 \mathrm{D}$ supergravity, such a construction would require [35, 22] going beyond the orbifold supergravity tensor calculus of [20, 21] where odd supergravity submultiplets (like our extrinsic curvature multiplet (3.30) and "odd·odd" terms in even multiplets are discarded.

${ }^{14}$ In our conventions, $R(\widehat{\omega})=e_{B}{ }^{M} e_{A}{ }^{N} R(\widehat{\omega})_{M N}{ }^{A B}$ with $R(\widehat{\omega})_{M N}{ }^{A B}=\partial_{M} \widehat{\omega}_{N}{ }^{A B}+\widehat{\omega}_{M}{ }^{A C} \widehat{\omega}_{N C}{ }^{B}-(M \leftrightarrow N)$, and $\psi_{M N}=D_{M}(\widehat{\omega}) \psi_{N}-D_{N}(\widehat{\omega}) \psi_{M}$.
} 
separately susy boundary action that removes the term linear in $S$. We add the action given in (3.38). The resulting improved bulk-plus-boundary supergravity action reads ${ }^{15}$

$$
\begin{gathered}
S_{S G}^{\mathrm{impr}}=\int_{\mathcal{M}} d^{3} x e_{3}\left[\frac{1}{2} R(\widehat{\omega})+\frac{1}{2} \bar{\psi}_{M} \gamma^{M N K} D(\widehat{\omega})_{N} \psi_{K}+\frac{1}{4} S^{2}\right] \\
+\int_{\partial \mathcal{M}} d^{2} x e_{2}\left(\widehat{K}+\frac{1}{2} \bar{\psi}_{a+} \gamma^{a} \gamma^{b} \psi_{b-}\right)
\end{gathered}
$$

where $\widehat{K}=e^{m a} \widehat{K}_{m a}$ with $\widehat{K}_{m a}=\widehat{\omega}_{m a \hat{3}}-\frac{1}{2} \bar{\psi}_{m+} \psi_{a-}$ which is the (symmetric) supercovariant extrinsic curvature tensor. The boundary term, which is obviously a susy generalization of the York-Gibbons-Hawking term [26], can also be written as follows

$$
\int_{\partial \mathcal{M}} d^{2} x e_{2}\left(\widetilde{K}+\frac{1}{2} \bar{\psi}_{a+} \gamma^{a b} \psi_{b-}\right)
$$

where $\widetilde{K}=e^{m a} \widetilde{K}_{m a}$ with $\widetilde{K}_{m a}=\widehat{\omega}_{m a \hat{3}}$ which is neither symmetric nor supercovariant under the (modified) $\epsilon_{+}$susy. The Euler-Lagrange variation of the improved supergravity action gives rise to the following boundary term,

$$
\int_{\partial \mathcal{M}} d^{2} x e_{2}\left[\delta e^{m a}\left(\widetilde{K}_{m a}-e_{m a} \widetilde{K}\right)+\delta \bar{\psi}_{m+} \gamma^{a b} \psi_{b-} e_{a}^{m}\right]
$$

Therefore, removing the term linear in $S$ in the boundary action of (4.12) by adding a separately susy boundary action (3.38) has improved the variational principle it two ways. First, the unacceptable boundary condition $e_{2}=0$ is avoided. Second, the boundary part of the Euler-Lagrange variation (known also as "the boundary field equation") is now in the " $p \delta q$ " form (by analogy with the Hamiltonian formulation). This allows one to derive "natural" boundary conditions (for on-shell fields) by requiring that the boundary variation vanishes for arbitrary $\delta q$ [36]. In our case, the role of " $q$ " is played by the induced supergravity multiplet $\left(e_{m}{ }^{a}, \psi_{m+}\right)$ of $(3.20)$.

It is very important for extending our construction to higher dimensions (where the full set of auxiliary fields is not always known or does not exist) that it is possible to eliminate the auxiliary field $S$ by its equation of motion $S=0$ while preserving susy of the action without the use of any boundary conditions. This indicates, for example, that even though there is no (off-shell) tensor calculus for 11D supergravity, the construction of Moss [18 can, perhaps, be improved so that susy of the 11D Horava-Witten action on the manifold with boundary does not require any boundary conditions on fields.

\footnotetext{
${ }^{15}$ The boundary term of the improved supergravity action (4.13) has the same form as the one found by Moss [18]. (Note that $2 \bar{\psi}_{a+} \gamma^{a} \gamma^{b} \psi_{b-}=\bar{\psi}_{a} \gamma^{a} \gamma^{b} \psi_{b}$.) However, there are essential differences. Moss uses an "adaptive coordinate system $e_{\hat{N} I}=\delta_{N I}$," which in our case would mean $e_{m}^{\hat{3}}=0$ and $e_{3}{ }^{\hat{3}}=1$. Moreover, his expression for the supercovariant extrinsic curvature involves $\psi_{N}$ (our $\psi_{3}$ ) and, therefore, could be equivalent to our (3.11), which involves $\psi_{\hat{3}}$, only if, in addition, $e_{3}{ }^{a}=0$. Finally, in the approach of Moss, susy of the bulk-plus-boundary action is claimed only using the $\psi_{m-}=0$ boundary condition. Our tensor calculus approach, on the other hand, leads to bulk-plus-boundary actions that are susy without using any boundary conditions.
} 
It is also instructive to find an alternative form of our bulk-plus-boundary action (4.13) by separating the fermionic bilinear parts in $\widehat{\omega}_{M A B}$ and $\widehat{K}$. Setting $S=0$, we obtain 12

$$
\begin{gathered}
\widetilde{S}_{S G}=\int_{\mathcal{M}} d^{3} x e_{3}\left[\frac{1}{2} R(\omega)+\frac{1}{2} \bar{\psi}_{M} \gamma^{M N K} D(\omega)_{N} \psi_{K}+O\left(\psi^{4}\right)\right] \\
+\int_{\partial \mathcal{M}} d^{2} x e_{2}\left(K+\frac{1}{2} \bar{\psi}_{a+} \gamma^{a b} \psi_{b-}\right)
\end{gathered}
$$

where $K$ is the standard bosonic extrinsic curvature term. In this form, ignoring the 4-fermi terms, the 3D bulk-plus-boundary action for supergravity was first found by Luckock and Moss in [37. ${ }^{16}$ We have determined all 4 -fermi terms in the bulk and boundary actions. We found 4-fermi terms in the bulk action which agree with the literature of supergravity, but no 4 -fermi terms on the boundary. So, the 2-fermi terms of [37] give already the complete boundary action. The new result of our construction is that the same boundary action is sufficient for "susy without BC" of the total bulk-plus-boundary action.

\section{Summary and Conclusions}

In this article, we have studied the issue of constructing locally susy bulk-plus-boundary actions in the simple setting of 3D $N=1$ supergravity. We demonstrated that the tensor calculus for 3D $N=1$ supergravity can be naturally extended to take boundaries into account. For a $3 \mathrm{D}$ scalar multiplet $(A, \chi, F)$, our " $F+A$ " formula (4.5) gives a bulk-plus-boundary action

$$
S_{F+A}=\int_{\mathcal{M}} d^{3} x e_{3}[F+\ldots]-\int_{\partial \mathcal{M}} d^{2} x e_{2} A
$$

which is "susy without BC" (its susy variation vanishes without the need to impose any BC on fields) under the half of bulk susy parametrized by $\epsilon_{+}$(satisfying $\gamma^{\hat{3}} \epsilon_{+}=\epsilon_{+}$when the Lorentz gauge (2.7) is imposed). Quite remarkably, this simple extension of the standard $F$ density formula works in $4 \mathrm{D} N=1$ sugra as well (where the $D$-density can also be similarly extended) [12].

The " $F+A$ " (extended $F$-density) formula can be applied to a variety of models. As an illustration, we applied it to the $3 \mathrm{D} N=1$ scalar curvature multiplet. The resulting bulkplus-boundary action (4.12) has the standard 3D $N=1$ sugra in the bulk and just the term $e_{2} S$ on the boundary. It is "susy without BC" by construction, but the field equation for the bulk auxiliary field $S$ gives not only $S=0$ in the bulk but also $e_{2}=0$ on the boundary, which is unacceptable. To resolve this problem while maintaining the "susy without BC" property, we looked for an additional separately susy boundary action containing the same term $e_{2} S$. The simplest such action is (3.38). Adding it to the minimal bulk-plus-boundary action given by the " $F+A$ " formula, we find that the $S$-term gets replaced by the York-Gibbons-Hawking

\footnotetext{
${ }^{16}$ In $5 \mathrm{D}$, the analog of this action was found in [19 and its "susy without BC" was established up to the 4 -fermi terms and terms involving the $5 \mathrm{D}$ graviphoton.
} 
extrinsic curvature term $K$ together with the gravitino bilinear $\bar{\psi}_{a+} \gamma^{a b} \psi_{b-}$. Neither the bulk nor the boundary action is separately susy, but their sum is and it is "susy without BC."

In order to construct separately susy boundary actions systematically, we have developed a co-dimension one decomposition of bulk supermultiplets. We found that the $3 \mathrm{D} N=1$ sugra multiplet $\left(e_{M}^{A}, \psi_{M}, S\right)$ decomposes into several 2D $N=(1,0)$ multiplets: the induced sugra multiplet $\left(e_{m}{ }^{a}, \psi_{m+}\right)$, the radion multiplet $\left(e_{3}{ }^{\hat{}},-\psi_{3-}+e_{3}{ }^{a} \psi_{a-}\right)$ and an "off-diagonal multiplet" $\left(e_{3 a},-e_{3}{ }^{3} \psi_{a-}+\gamma_{a} \psi_{3+}\right)$ [12]. (The other off-diagonal component of the vielbein, $e_{m}{ }^{\hat{3}}$, vanishes in our Lorentz gauge (2.7).) With the parity assignments given in (3.7), the first two multiplets are "even" and the last one is "odd." The $3 \mathrm{D} N=1$ scalar multiplet $(A, \chi, F)$ allows a similar decomposition; see (3.33). Explicit verification that these submultiplets transform as standard 2D $N=(1,0)$ supermultiplets is tedious [12], but our analysis of the gauge algebra guarantees that the co-dimension one decomposition does work and does not require any (boundary) conditions on fields.

In the superspace formulation, one can act on superfields with superspace covariant derivatives to construct new superfields. In the tensor calculus, the new multiplets can be constructed simply by choosing an appropriate lowest component. For example, starting with $\gamma^{a} \psi_{a-}$, we obtain our extrinsic curvature (scalar) multiplet (3.30). Starting with $\psi_{a-}$, we similarly obtain an extrinsic curvature tensor multiplet [12]. The multiplets obtained in this way can, together with any number of independent boundary matter multiplets, be used to construct separately susy boundary actions using the standard 2D $N=(1,0) F$-density formula (3.35). In conjunction with our " $F+A$ " formula, this gives the most general bulkplus-boundary actions that are "susy without BC." However, requiring that the variational principle yields field equations that are not too strong restricts the choice of boundary actions that one can allow [12].

We should note that the Lorentz gauge (2.7) that we used in this work allows a tremendous simplification of the algebra. At the same time, our results can be extended to the case when no Lorentz gauge is imposed (see e.g. footnote 12) 12. We also note that our tensor calculus approach relies heavily on the off-shell supergravity formulation (with auxiliary fields). Such a formulation is not always available in higher dimensions. Nonetheless, a concrete higher dimensional model (such as the 11D Horava-Witten construction) has still a chance to be "susy without BC" as we discussed in section 4.3.

Our program of "susy without BC" can and should be extended to (a) dimensions higher than three, (b) the superspace formulation, (c) superconformal symmetries and superconformal actions, (d) BRST symmetry. Some progress in these directions has already been achieved [12]. Ultimately, this would allow to have complete control over the models discussed in the Introduction as well as other models where symmetries and boundaries collide.

Acknowledgments. We would like to thank Dima Vassilevich for his participation in the beginning of this project. D.V.B. also thanks Jon Bagger for discussions on related 
topics. We thank the C. N. Yang Institute for Theoretical Physics at SUNY Stony Brook and Deutsches Electronen-Synchrotron DESY in Hamburg for hospitality extended to us during visits related to this project. The research of D.V.B. was supported in part by the German Science Foundation (DFG). The research of P.v.N. was supported by the NSF grant no. PHY-0354776.

\section{References}

[1] J. Wess and B. Zumino, Nucl. Phys. B 78, 1 (1974).

[2] S. Ferrara and P. van Nieuwenhuizen, Phys. Lett. B 76, 404 (1978); Phys. Lett. B 78, 573 (1978).

[3] K. S. Stelle and P. C. West, Phys. Lett. B 77, 376 (1978); Nucl. Phys. B 145, 175 (1978).

[4] A. Salam and J. A. Strathdee, Nucl. Phys. B 76, 477 (1974); Phys. Rev. D 11, 1521 (1975).

[5] J. Wess and B. Zumino, Phys. Lett. B 66, 361 (1977).

[6] J. Wess and B. Zumino, Phys. Lett. B 79, 394 (1978).

[7] P. Di Vecchia, B. Durhuus, P. Olesen and J. L. Petersen, Nucl. Phys. B 207, 77 (1982); Nucl. Phys. B 217, 395 (1983).

[8] Y. Igarashi, Phys. Rev. D 30, 1812 (1984); Y. Igarashi and T. Nonoyama, Phys. Lett. B 161, 103 (1985); Phys. Rev. D 34 (1986) 1928.

[9] S. W. Hawking, Phys. Lett. B 126, 175 (1983); A. K. Chatterjee and P. Majumdar, Phys. Lett. B 159, 37 (1985); P. D. D’Eath, Nucl. Phys. B 269, 665 (1986); S. Elitzur, G. W. Moore, A. Schwimmer and N. Seiberg, Nucl. Phys. B 326, 108 (1989); N. Sakai and Y. Tanii, Prog. Theor. Phys. 83, 968 (1990); H. Luckock, Annals Phys. 194, 113 (1989); J. Phys. A 24, L1057 (1991); N. P. Warner, Nucl. Phys. B 450, 663 (1995); T. Inami, S. Odake and Y. Z. Zhang, Phys. Lett. B 359, 118 (1995); G. Esposito and A. Y. Kamenshchik, Phys. Rev. D 54, 3869 (1996); C. Albertsson, U. Lindstrom and M. Zabzine, Commun. Math. Phys. 233, 403 (2003); Nucl. Phys. B 678, 295 (2004); U. Lindstrom and M. Zabzine, Phys. Lett. B 560, 108 (2003); P. Koerber, S. Nevens and A. Sevrin, JHEP 0311, 066 (2003); S. F. Hassan, arXiv:hep-th/0308201; I. V. Melnikov, M. R. Plesser and S. Rinke, arXiv:hep-th/0309223.

[10] U. Lindstrom, M. Rocek and P. van Nieuwenhuizen, Nucl. Phys. B 662, 147 (2003).

[11] P. van Nieuwenhuizen and D. V. Vassilevich, Class. Quant. Grav. 22, 5029 (2005).

[12] D. V. Belyaev and P. van Nieuwenhuizen, to appear.

[13] D. V. Belyaev, JHEP 0601, 046 (2006).

[14] N. Marcus, A. Sagnotti and W. Siegel, Nucl. Phys. B 224, 159 (1983); E. A. Mirabelli and M. E. Peskin, Phys. Rev. D 58, 065002 (1998); N. Arkani-Hamed, T. Gregoire and J. G. Wacker, JHEP 0203, 055 (2002); Y. Sakamura, Nucl. Phys. B 656, 132 (2003).

[15] P. Horava and E. Witten, Nucl. Phys. B 460, 506 (1996); Nucl. Phys. B 475, 94 (1996).

[16] L. Randall and R. Sundrum, Phys. Rev. Lett. 83, 3370 (1999); Phys. Rev. Lett. 83, 4690 (1999). 
[17] R. Altendorfer, J. Bagger and D. Nemeschansky, Phys. Rev. D 63, 125025 (2001);

T. Gherghetta and A. Pomarol, Nucl. Phys. B 586, 141 (2000); A. Falkowski, Z. Lalak and S. Pokorski, Phys. Lett. B 491, 172 (2000); E. Bergshoeff, R. Kallosh and A. Van Proeyen, JHEP 0010, 033 (2000).

[18] I. G. Moss, Phys. Lett. B 577, 71 (2003); Nucl. Phys. B 729, 179 (2005).

[19] D. V. Belyaev, JHEP 0601, 047 (2006).

[20] M. Zucker, Phys. Rev. D 64, 024024 (2001); Fortsch. Phys. 51, 899 (2003).

[21] T. Kugo and K. Ohashi, Prog. Theor. Phys. 108, 203 (2002).

[22] D. V. Belyaev, arXiv:0710.4540 [hep-th].

[23] J. Bagger and D. V. Belyaev, Phys. Rev. D 67, 025004 (2003); JHEP 0306, 013 (2003); Phys. Rev. D 72, 065007 (2005); A. Falkowski, JHEP 0505, 073 (2005).

[24] T. Uematsu, Z. Phys. C 29, 143 (1985); Z. Phys. C 32, 33 (1986).

[25] T. Uematsu, Phys. Lett. B 183, 304 (1987).

[26] J. W. York, Jr., Phys. Rev. Lett. 28, 1082 (1972); G. W. Gibbons and S. W. Hawking, Phys. Rev. D 15, 2752 (1977).

[27] D. Z. Freedman and P. van Nieuwenhuizen, Phys. Rev. D 14, 912 (1976).

[28] J. F. Luciani, Nucl. Phys. B 135, 111 (1978); A. Salam and J. A. Strathdee, Annals Phys. 141, 316 (1982).

[29] J. Schwinger, Phys. Rev. 130, 1253 (1963).

[30] T. W. B. Kibble, J. Math. Phys. 4, 1433 (1963).

[31] S. Deser, J. H. Kay and K. S. Stelle, Phys. Rev. D 16, 2448 (1977).

[32] J. Isenberg and J. M. Nester, "Canonical Gravity," in General relativity and gravitation, ed. A. Held, Plenum Press, New York, 1980.

[33] C. Csaki, M. Graesser, L. Randall and J. Terning, Phys. Rev. D 62, 045015 (2000).

[34] W. D. Linch III, M. A. Luty and J. Phillips, Phys. Rev. D 68, 025008 (2003).

[35] F. Paccetti Correia, M. G. Schmidt and Z. Tavartkiladze, Nucl. Phys. B 709, 141 (2005).

[36] R. Courant, Bull. Amer. Math. Soc. 49, 1 (1943); N. H. Barth, Class. Quant. Grav. 2, 497 (1985).

[37] H. Luckock and I. Moss, Class. Quant. Grav. 6, 1993 (1989). 